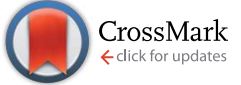

Cite this: Chem. Sci., 2014, 5, 3563

\title{
Uncovering the origin of Z-configured double bonds in polyketides: intermediate $E$-double bond formation during borrelidin biosynthesis $\uparrow$
}

\author{
Nadine Kandziora, ${ }^{a}$ Jennifer N. Andexer, ${ }^{\text {bc }}$ Steven J. Moss, ${ }^{d}$ Barrie Wilkinson, ${ }^{e}$ \\ Peter F. Leadlay ${ }^{b}$ and Frank Hahn*ab
}

Formation of $Z$-configured double bonds in reduced polyketides is uncommon and their origins have not been extensively studied. To investigate the origin of the $Z$-configured double bond in the macrolide borrelidin, the recombinant dehydratase domains $\mathrm{BorDH} 2$ and $\mathrm{BorDH} 3$ were assayed with a synthetic analogue of the predicted tetraketide substrate. The configuration of the dehydrated products was determined to be $E$ in both cases by comparison to synthetic standards. Detailed NMR spectroscopic analysis of the biosynthetic intermediate pre-borrelidin confirmed the E,E-configuration of the fulllength polyketide synthase product. In contrast to a previously-proposed hypothesis, our results show that in this case the $Z$-configured double bond is not formed via dehydration from a 3 L-configured precursor, but rather as the result of a later isomerization process.

Received 25th March 2014

Accepted 17th June 2014

DOI: $10.1039 / c 4 s c 00883 a$

www.rsc.org/chemicalscience

$E$-configuration. It has been proposed that $E$-double bonds arise from DH-catalysed syn-dehydration of 3-D (or 3-R) hydroxythioesters and that the rare $Z$-configured double bonds originate from 3-L (or 3-S) precursors. ${ }^{2 a}$ The configuration of this precursor is determined by the preceding KR. Characteristic active site residues in KRs have been identified that directly influence the stereochemical outcome of the reduction on C-3 as well as on $\mathrm{C}-2 .{ }^{2}$ However, there are individual modules (for example in the PKSs for rifamycin, borrelidin, halstoctacosanolide, thuggacin and phoslactomycin) where this rule does not seem to apply. In these cases $Z$-configured double bonds are present in the final natural product even though the configuration of the $\mathrm{DH}$ precursors in the particular modules is predicted to be 3-D according to the active site residues in the preceding KR. ${ }^{3}$

Although double bonds are important and abundant structural features of reduced polyketides, their DH-catalysed formation has not been intensively studied and only little is known about the stereoselectivity and substrate specificity of these domains. We and others have previously shown that $\mathrm{DH}$ domains may retain catalytic competence as isolated domains ${ }^{a}$ Institut für Organische Chemie, Leibniz Universität Hannover, Schneiderberg 1 B, 30167 Hannover, Germany. E-mail: frank.hahn@oci.uni-hannover.de; Fax: +49 511762-3011; Tel: +49 511-762-4603

${ }^{b}$ Department of Biochemistry, University of Cambridge (UK), 80 Tennis Court Road, Cambridge CB2 1QW, UK

'Institut für Pharmazeutische Wissenschaften, Albert-Ludwigs-Universität Freiburg, Albertstr. 25, 79104 Freiburg im Breisgau, Germany

${ }^{d}$ Isomerase Therapeutics, Chesterford Research Park, Cambridge CB10 1XL, UK ${ }^{e}$ Department of Molecular Microbiology, John Innes Centre Norwich NR4 7UH, UK $\dagger$ Electronic supplementary information (ESI) available: Experimental procedures, characterizytion data and spectra of synthesized surrogate molecules. Procedures for enzyme assays and characterization data of assay products and pre-borrelidin. See DOI: $10.1039 / \mathrm{c} 4 \mathrm{sc} 00883 \mathrm{a}$

in vitro. ${ }^{4} \mathrm{X}$-ray crystal structures have been obtained for

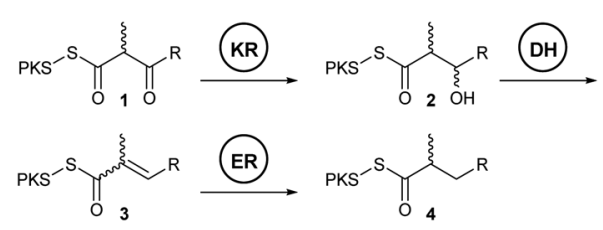

Scheme 1 Processing of PKS intermediates by reductive loop domains. ${ }^{1}$ 
$E$-selective DH domains from the curacin and the erythromycin pathway; ${ }^{5} E$-selective $\mathrm{DH}$ domains from the erythromycin and the nanchangmycin pathway have been studied in vitro. ${ }^{4 a, b}$

Much less is known about putatively $Z$-selective $\mathrm{DH}$ domains. Of the above-mentioned exceptional cases, only the $\mathrm{DH}$ domains from the respective modules of the rifamycin and the borrelidin PKS have been studied in vitro so far. ${ }^{4 c, \boldsymbol{d}}$ However, in both cases diketide surrogates have been used which significantly differ from the natural precursors. Additionally, the X-ray crystal structure for RifDH10 from the rifamycin pathway has been reported. Recently, a TE(Fr9-TE2)-catalyzed dehydration reaction leading to the formation of a $Z$-configured double bond was observed in the biosynthesis of the spliceosome inhibitor FR901464 and reconstituted in vitro. ${ }^{4 e} \mathrm{TE}$ domains could be classified into the same hotdog superfamily as DH domains and therefore they show structural similarity to each other. Further studies will however have to clarify if mechanistic analogies exist between both domains as well.

The macrolide borrelidin (10) has promising antibacterial, antimalarial and anticancer activities. It acts as an angiogenesis inhibitor by affecting threonyl-tRNA synthetase and the spliceosome-associated protein formin binding protein 21 , thereby acting as a modulator of alternative splicing. It contains several unusual structural features, including a cyclopentanecarboxylic acid moiety, which derives from a $(1 R, 2 R)$-cyclopentanedicarboxylic acid starter unit, and a carbonitrile substituent at C-12 (Fig. 1a). ${ }^{6}$ Total synthesis and X-ray diffraction analysis have confirmed the overall structure of borrelidin including the $(Z, E)$-configuration of the conjugated cyanodiene in the southern fragment. ${ }^{7}$

These two double bonds are formed during biosynthesis by the consecutive action of PKS extension modules two and three
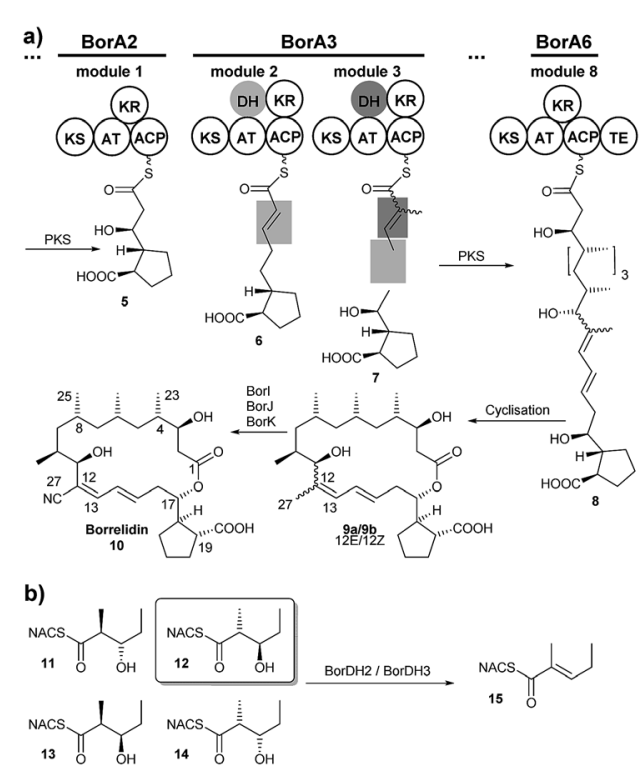

Fig. 1 (a) Borrelidin is biosynthesized on a modular PKS-I. The DH domains of modules 2 and 3 are highlighted in light and dark grey. (b) Previous study on recombinant BorDH2 and BorDH3. Both $\mathrm{DH}$ domains converted only the 2-D,3-D isomer of 3-hydroxy-2-methylSNAC-pentanoate, and the product in each case contained an $E$-configured double bond. HSNAC: $N$-acetylcysteamine. ${ }^{4 c, 8}$
(Fig. 1a). ${ }^{8}$ In both modules the KR domains bear the characteristic LDD-motif in their active sites, which predicts a 3-Dconfiguration in the hydroxyacyl DH-precursors. ${ }^{2 a, b}$ Interpretation of the BorKR3 sequence according to a recently published algorithm furthermore points towards a D-configuration at the branched 2-position of the BorDH3 precursor. ${ }^{2 c}$ Based on these analyses the configuration of the borrelidin diene is predicted to be $E, E$ and not $Z, E$. This conflict between predicted and confirmed structure makes borrelidin an attractive model system to study the role of PKS-DH domains in the formation of rare $Z$-configured double bonds. Either the BorDH3 represents an exception to the proposed rule, or the $Z$-configured double bond must be established at a later stage in the pathway. ${ }^{4 c}$ One of the most direct and feasible ways to answer this question would be an in vitro experiment with isolated enzymes and a realistic substrate surrogate.

In a previous study we demonstrated the catalytic activity of isolated recombinant BorDH2 and BorDH3 on simple 3-hydroxy-2-methyl-SNAC-pentanoate substrate surrogates in vitro (SNAC: $N$-acetylcysteamine). ${ }^{4 c}$ We found that both domains behave similarly in that they selectively convert only the 2-D,3-D precursor 12 and produce only the E-configured product 15 (Fig. 1b). While this result is consistent with syn-dehydration, assuming that the substrate backbone is similarly positioned as in porcine fatty acid synthase-DH, curacin-DHs and erythromycin-DH, it leaves open the question whether the $Z$-configured double bond in borrelidin is actually installed by a biosynthetic process that occurs after polyketide chain assembly or if the outcome of the in vitro experiment with BorDH3 was biased by the use of relatively simple model substrates.

It is known from in vitro experiments with other isolated PKS domains that the substrate structure and the nature of the thioester exert a crucial influence on the stereochemical course of the reaction. ${ }^{4 \boldsymbol{d}, 9}$ For RifDH10, it was observed that the stereospecificity of the dehydration reaction changes if the hydroxyacyl substrate is attached either to an ACP other than the natural one or to truncated surrogates of the ACP-bound form like SNAC or pantetheine. In the latter cases, the preference switches completely from the native $(2 S, 3 S)$ precursor to the enantiomeric $(2 R, 3 R)$. Nevertheless, in agreement with a syndehydration the $E$-selectivity of the domain was not altered by these changes. We now report that further analysis of the BorDH2 and BorDH3 domains with a more realistic substrate has substantiated our previous results, allowed us to study the influence of the substrate structure and better to understand the biosynthetic origin of the $(Z, E)$-diene.

\section{Results and discussion}

We synthesised the tetraketide precursors $17 \mathbf{a}$ and $17 \mathbf{b}$ as a mixture of both 2,3-anti-stereoisomers in the form of their SNAC-thioesters, as well as reference molecules 18, 19 and 20 (Scheme 2). ${ }^{10}$ All synthetic routes diverged from a common, $\alpha, \beta-$ unsaturated precursor aldehyde, which was obtained by metathesis reaction of olefin 16 with crotonaldehyde and Second Generation Grubbs catalyst. The DH substrates 17a and 17b were obtained by an anti-selective boron aldol reaction, 


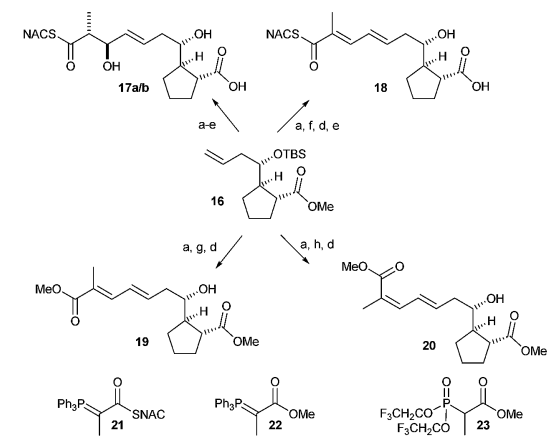

Scheme 2 Synthesis of precursors and reference molecules. (a) Crotonaldehyde, Second Generation Grubbs catalyst, $\mathrm{CH}_{2} \mathrm{Cl}_{2}, 40{ }^{\circ} \mathrm{C}$, $2 \mathrm{~h}$; (b) $\mathrm{PhSCOC}_{2} \mathrm{H}_{5}, \mathrm{Cy}_{2} \mathrm{BCl}, \mathrm{NMe}_{2} \mathrm{Et}_{1} \mathrm{Et}_{2} \mathrm{O}$ then aldehyde in $\mathrm{Et}_{2} \mathrm{O}$, $-78{ }^{\circ} \mathrm{C}$ to $-30{ }^{\circ} \mathrm{C}, 16 \mathrm{~h}, 57 \%$ over two steps; (c) HSNAC, DIPEA, DMF, r.t., 16 h, 70\%; (d) THF : $\mathrm{H}_{2} \mathrm{O}: \mathrm{FA}(6: 3: 1)$, r.t., 2 days; (e) pig liver esterase, phosphate buffer, r.t., 2-5 days; (f) $21, \mathrm{CH}_{2} \mathrm{Cl}_{2}, 50{ }^{\circ} \mathrm{C}, 16 \mathrm{~h}$, $88 \%$ over two steps; (g) $22, \mathrm{CH}_{2} \mathrm{Cl}_{2}, 50{ }^{\circ} \mathrm{C}, 21 \mathrm{~h}, 64 \%$ over two steps; (h) 23,18 -crown- $6, \mathrm{~K}_{2} \mathrm{CO}_{3}, \mathrm{THF},-20{ }^{\circ} \mathrm{C}$ to $0{ }^{\circ} \mathrm{C}, 5 \mathrm{~h}, 47 \%$ over two steps. Overall yields: $32 \%$ over five steps for $17 \mathrm{a} / 17 \mathrm{~b}, 59 \%$ over four steps for $18,40 \%$ over three steps for $19,28 \%$ over three steps for 20 . Cy: cyclohexyl, FA: formic acid. ${ }^{10}$

followed by thiol exchange and ensuing acidic deprotection of the TBS ether and esterase-catalysed cleavage of the methyl ester. The SNAC thioester of the $E, E$-configured product 18 was obtained by Wittig reaction with phosphorane 21 and deprotection following the protocol established for $\mathbf{1 7 a}$ and $\mathbf{1 7} \mathbf{b}$. The isomeric dienes 19 and 20 in the form of their bis-methyl esters were accessed via Wittig reaction with stabilised phosphorane 22 and Still-Gennari olefination with phosphonate 23, respectively, followed by TBS deprotection.

The mixture of the two anti-2-methyl-3-hydroxy thioesters 17a and 17b was incubated with recombinant BorDH2 and BorDH3, respectively. After $16 \mathrm{~h}$, the reaction was terminated by extraction of the organic materials with ethyl acetate. Direct analysis of this crude assay extract showed conversion of the starting material into a dehydrated compound (Fig. $2 \mathrm{c}$ and d). Enzyme-free control reactions, which were treated under identical conditions, reproducibly showed no conversion of $17 \mathbf{a}$ and 17b into the dehydrated product (Fig. 2a). To rule out the possibility of catalysis by a contaminant that was purified along with the $\mathrm{DH}$, the experiment was repeated with a purified mutant of BorDH3 in which the active site histidine had been exchanged to an alanine (Fig. 2b). No conversion was obtained in this case, confirming that the recombinant borrelidin DHs were responsible for the observed dehydration.

In order to establish the configuration of the newly formed double bond by comparison to the reference molecules 19 and 20, we derivatized the crude assay product mixture. Saponification with $1 \mathrm{M} \mathrm{NaOH}$ followed by methylation with trimethylsilyl diazomethane gave bis-methyl esters, which were analysed by UPLC-MS (Fig. 3a, S3 and S4†). Comparison to the synthetic reference molecules established the configuration of the products to be $E, E$. A similar result was obtained when BorDH2 was employed instead of BorDH3, showing that this domain also retains activity and its natural stereoselectivity
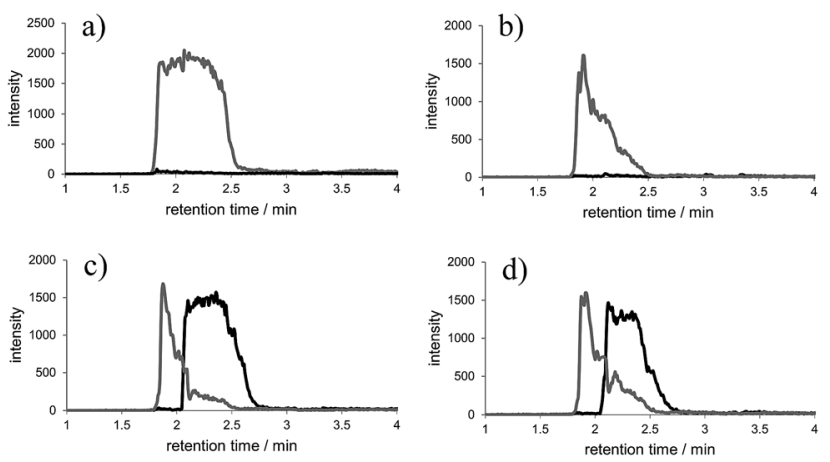

Fig. 2 UPLC-MS analysis of DH assays. The grey line represents the hydrated starting material $\left(17 \mathrm{a} / 17 \mathrm{~b}:[\mathrm{M}+\mathrm{Na}]^{+}=410\right)$, the black line corresponds to the dehydration product $\left(18:[\mathrm{M}+\mathrm{Na}]^{+}=392\right)$. The starting material is stable towards dehydration in an enzyme-free overnight incubation as well as towards reaction with a deactivated active-site mutant (a) and (b). It shows dehydration only after incubation with the wild-type BorDH domains (c) and (d). (a) Enzyme-free overnight incubation of $17 a / 17 b$; (b) overnight incubation of $17 a / 17 b$ with BorDH3-H49A; (c) overnight incubation of 17a/17b with BorDH2; (d) overnight incubation of $17 \mathrm{a} / 17 \mathrm{~b}$ with BorDH3.

when presented with a substrate of larger size than the natural one.

To exclude the possibility of double bond isomerization during derivatization, we repeated the experiment with BorDH3 on a larger scale and analysed the product by ${ }^{1} \mathrm{H}$ NMR spectroscopy. $10 \mathrm{mg}$ of the mix of $\mathbf{1 7 a}$ and $\mathbf{1 7} \mathbf{b}$ were incubated with BorDH3 for $16 \mathrm{~h}$ at $37^{\circ} \mathrm{C}$. After extraction into organic solvent, the crude material was directly analyzed by ${ }^{1} \mathrm{H}$ NMR. The resulting ${ }^{1} \mathrm{H}$ NMR spectrum was in full agreement with a partial conversion of $\mathbf{1 7 a / 1 7 b}$ into $\mathbf{1 8}$, as judged by comparison to a synthetic sample of $\mathbf{1 8}$ (Fig. $3 \mathrm{~b}$ and $\mathrm{S} 5 \dagger$ ).

Additional NOE experiments were conducted to confirm the $(2 E, 4 E)$-configuration of the BorDH3 assay product (Fig. 3c). While irradiation at $7.23 \mathrm{ppm}(\mathrm{H} 3)$ showed a correlation to $\mathrm{H} 5$, but not to $2-\mathrm{CH}_{3}$, irradiation at $6.57 \mathrm{ppm}(\mathrm{H} 4)$ exclusively showed correlation to $2-\mathrm{CH}_{3}$. This is in full agreement to a syn relationship of the 2- $\mathrm{CH}_{3}$ group and the vinyl fragment along the $\mathrm{C} 2-\mathrm{C} 3$ olefin and therefore a $(2 E, 4 E)$-configuration of the diene.

From these results, we conclude that BorDH2 and more importantly BorDH3 each selectively transform the tetraketide precursor into an $(2 E, 4 E)$-diene. Accordingly, the isomerization of the double bond must occur at a later stage in biosynthesis.

This result prompted us to examine in detail the configuration of the C12-C13 double bond in 12-desnitrile-12-methylborrelidin (9a/9b). This metabolite (pre-borrelidin) was originally isolated as the product of the mutant strain $\Delta$ borI, which is devoid of BorI. ${ }^{8 b}$ BorI catalyses the oxidation of the allylic position C27 in one of the potential precursors $9 a$ or $9 \mathbf{b}$ to activate it for transamination and further oxidation/dehydration to the carbonitrile by BorJ and (probably) BorK (Fig. 1a). Previous biotransformation experiments confirmed that preborrelidin is indeed a competent intermediate of the pathway and not a shunt product. ${ }^{8 b}$

Initial NMR analysis using ${ }^{1} \mathrm{H},{ }^{13} \mathrm{C}$, DEPT, COSY, HSQC and HMBC experiments had shown that pre-borrelidin constitutionally 

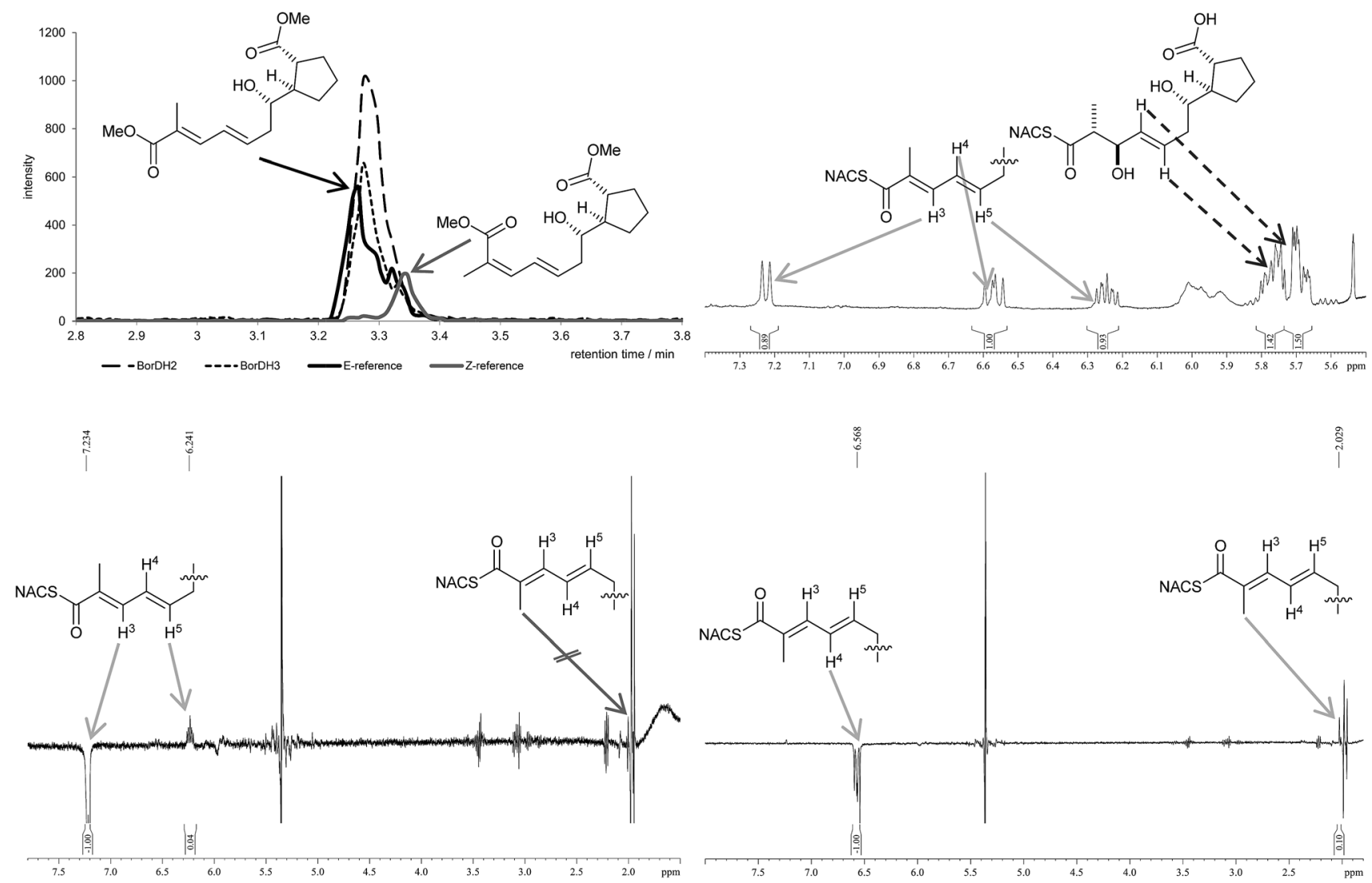

Fig. 3 (a) UPLC-MS analysis of derivatized assay products and comparison to reference molecules; grey line: (Z)-isomer, reference bis-methylester 20; black line: $(E)$-isomer, reference bis-methylester 19; dashed line: derivatized dehydration product of BorDH2; dotted line: derivatized dehydration product of BorDH3; (b) expansion of the olefinic region in the ${ }^{1} \mathrm{H} N M R$ spectrum of the BorDH3 assay product in $\mathrm{d}_{2}$-dichloromethane; (c) NOE analysis of the BorDH3 assay product. Upon irradiation at $7.23 \mathrm{ppm}(\mathrm{H} 3)$, correlation to $\mathrm{H} 5(6.24 \mathrm{ppm})$, but not to $2-\mathrm{CH}_{3}(2.03 \mathrm{ppm})$ was observed; (d) NOE analysis of the BorDH3 assay product. Irradiation at $6.57 \mathrm{ppm}(\mathrm{H} 4)$ exclusively showed correlation to 2- $\mathrm{CH}_{3}$.

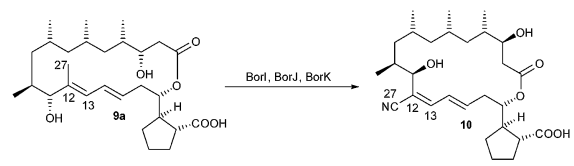

Fig. 4 Proposed final steps of borrelidin biosynthesis. The formation of the carbonitrile is proposed to occur via oxidation of pre-borrelidin 9a at C27 to an allylic aldehyde (catalysed by Borl), transamination (BorJ) and further oxidation (possibly BorK). ${ }^{8 b}$

differs from borrelidin only in bearing a methyl group instead of a carbonitrile at $\mathrm{C} 27 .^{8 b}$ We have confirmed these findings and have now carried out additional NOESY and NOE experiments (see ESI, Fig. S7 and S8†) which firmly establish that the C12-C13 double bond has exclusively the $E$-configuration. Therefore, pre-borrelidin has the $(12 E, 14 E)$-configuration shown for 9a (Fig. 1a and 4) and the isomerization to the $(12 Z, 14 E)$-configuration found in borrelidin 10 must occur at a later stage of the biosynthesis.

To exclude the possibility of isomerization having occurred during workup, the fermentation of $\Delta$ borI was monitored by HPLC-MS, which showed similar retention times for the product formed during fermentation and after final isolation. This result is in accordance with the conclusion from our in vitro experiments, that the diene is first formed exclusively in an $E, E$-fashion.

\section{Conclusions}

This study is the first example in which a potentially $Z$-selective PKS-DH domain was tested in vitro with a realistic substrate mimic that resembles the full polyketide portion of the biosynthetic intermediate. Although some $Z$-configured double bonds in reduced polyketides clearly are formed by syn dehydration of 3-L alcohols, our results reinforce the view that a subsequent isomerization or other biosynthetic process is likely to be responsible for their formation in many cases. ${ }^{4 e, 11}$

Our experiments also provide further valuable insight into enzymology of PKS domains. Together with previous reports, our assay results of BorDH2 and BorDH3 with such different substrate surrogates like 11-14, 17a and 17b suggest that PKSDH domains generally catalyse dehydrations with relaxed 
substrate specificity, but with high stereoselectivity with respect to the configuration of the double bond between $\mathrm{C} 2$ and $\mathrm{C} 3$. $^{4 \mathrm{a}-\mathrm{d}}$ This result is encouraging for their application in chemoenzymatic synthesis.

We can now assign the timing of the $E$ - $Z$-isomerization to the very final steps of borrelidin biosynthesis. Additional in vitro experiments with isolated enzymes will be necessary to elucidate the specific mechanism of isomerization and nitrile formation in the terminal steps of the borrelidin pathway.

\section{Acknowledgements}

We thank the Marie Curie programme of the European Union, the Emmy Noether programme of the Deutsche Forschungsgemeinschaft (Fellowships to F.H.) and the DAAD (Fellowship to J.N.A.) for financial support. We would also like to thank the NMR facility and the mass facility of the OCI Hanover as well as Prof. Andreas Kirschning and his group for general support and helpful discussion.

\section{Notes and references}

1 (a) J. Staunton and K. J. Weissman, Nat. Prod. Rep., 2001, 18, 380; (b) L. Kellenberger, I. S. Galloway, G. Sauter, G. Böhm, U. Hanefeld, J. Cortés, J. Staunton and P. F. Leadlay, ChemBioChem, 2008, 9, 2740; (c) D. H. Kwan, S. F. Haydock and P. F. Leadlay, Chem. Biol., 2008, 15, 1231; (d) A. T. Keatinge-Clay, Chem. Biol., 2007, 14, 898; (e) C. R. Valenzano, R. J. Lawson, A. Y. Chen, C. Khosla and D. E. Cane, J. Am. Chem. Soc., 2009, 131, 18501.

2 (a) R. Reid, M. Piagentini, E. Rodriguez, G. Ashley, N. Viswanathan, J. Carney, D. V. Santi, C. R. Hutchinson and R. McDaniel, Biochemistry, 2003, 42, 72; (b) P. Caffrey, ChemBioChem, 2003, 7, 654; (c) A. Kitsche and M. Kalesse, ChemBioChem, 2013, 14, 851.

3 (a) P. R. August, L. Tang, Y. J. Yoon, S. Ning, R. Müller, T.-W. Yu, M. Taylor, D. Hoffmann, C.-G. Kim, X. Zhang, C. R. Hutchinson and H. G. Floss, Chem. Biol., 1998, 5, 69; (b) S. Tohyama, K. Kakinuma and T. Eguchi, J. Antibiot., 2006, 59, 44; (c) M. Bock, K. Buntin, R. Müller and A. Kirschning, Angew. Chem., 2008, 120, 2341; Angew. Chem., Int. Ed., 2008, 47, 2308; (d) N. Palaniappan, M. M. Alhamadsheh and K. A. Reynolds, J. Am. Chem. Soc., 2008, 130, 12236.

4 (a) C. R. Valenzano, Y.-O. You, A. Garg, A. Keatinge-Clay, C. Khosla and D. E. Cane, J. Am. Chem. Soc., 2010, 132, 14697; (b) X. Guo, T. Liu, C. R. Valenzano, Z. Deng and D. E. Cane, J. Am. Chem. Soc., 2010, 132, 14694; (c) O. Vergnolle, F. Hahn, A. Baerga-Ortiz, P. F. Leadlay and J. N. Andexer, ChemBioChem, 2011, 12, 1011; (d) D. Gay, Y.-O. You, A. Keatinge-Clay and D. E. Cane, Biochemistry,
2013, 52, 8916; (e) H.-Y. He, M.-C. Tang, F. Zhang and G.-L. Tang, J. Am. Chem. Soc., 2014, 136, 4488.

5 (a) D. L. Akey, J. R. Razelun, J. Tehranisa, D. H. Sherman, W. H. Gerwick and J. L. Smith, Structure, 2010, 18, 94; (b) A. Keatinge-Clay, J. Mol. Biol., 2008, 384(4), 941.

6 (a) J. Berger, L. M. Jampolsky and M. Goldberg, Arch. Biochem., 1949, 22, 476; (b) W. Keller-Scheirlein, Helv. Chim. Acta, 1967, 50, 731; (c) K. Otoguro, H. Ui, A. Ishiyama, M. Kobayashi, H. Togashi, Y. Takahashi, R. Masuma, H. Tanaka, H. Tomoda, H. Yamada and S. Omura, J. Antibiot., 2003, 56, 727; (d) T. Kawamura, D. Liu, M. J. Towle, R. Kageyama, N. Tsukahara, T. Wakabayashi and B. A. Littlefield, J. Antibiot., 2003, 56, 709; (e) T. F. Williams, A. C. Mirando, B. Wilkinson, C. S. Francklyn and K. M. Lounsbury, Sci. Rep., 2013, 3, 1317; $(f)$ J. Woolard, W. Vousden, S. J. Moss, A. Krishnakumar, M. V. R. Gammons, D. G. Nowak, N. Dixon, J. Micklefield, A. Spannhoff, M. T. Bedford, M. A. Gregory, C. J. Martin, P. F. Leadlay, M. Q. Zhang, S. J. Harper, D. O. Bates and B. Wilkinson, Chem. Sci., 2011, 2, 273.

7 (a) T. Nagamitsu, D. Takano, T. Fukuda, K. Otoguro, I. Kuwajima, Y. Harigaya and S. Omura, Org. Lett., 2004, 6, 1865; (b) M. O. Duffey, A. LeTiran and J. P. Morken, J. Am. Chem. Soc., 2003, 125, 1458; (c) B. G. Vong, S. H. Kim, S. Abraham and E. A. Theodorakis, Angew. Chem., 2004, 116, 4037; Angew. Chem., Int. Ed., 2004, 43, 3947; (d) B. F. Anderson, A. J. Herlt, R. W. Rickards and G. B. Robertson, Aust. J. Chem., 1989, 42, 717.

8 (a) C. Olano, B. Wilkinson, C. Sánchez, S. J. Moss, R. Sheridan, V. Math, A. J. Weston, A. F. Braña, C. J. Martin, M. Oliynyk, C. Méndez, P. F. Leadlay and J. A. Salas, Chem. Biol., 2004, 11, 87; (b) C. Olano, S. J. Moss, A. F. Braña, R. M. Sheridan, V. Math, A. J. Weston, C. Méndez, P. F. Leadlay, B. Wilkinson and J. A. Salas, Mol. Microbiol., 2004, 6, 1745.

9 (a) A. P. Siskos, A. Baerga-Ortiz, S. Bali, V. Stein, H. Mamdani, D. Spiteller, B. Popovic, J. B. Spencer, J. Staunton, K. J. Weissman and P. F. Leadlay, Chem. Biol., 2005, 12, 1145; (b) S. Bali and K. J. Weissman, ChemBioChem, 2006, 7, 1935; (c) S. K. Piasecki, C. A. Taylor, J. F. Detelich, J. Liu, J. Zheng, A. Komsoukaniants, D. R. Siegel and A. T. Keatinge-Clay, Chem. Biol., 2011, 18, 1331; (d) M. Häckh, M. Müller and S. Lüdeke, Chem.-Eur. J., 2013, 19, 8922.

10 F. Hahn, N. Kandziora, S. Friedrich and P. F. Leadlay, Beilstein J. Org. Chem., 2014, 10, 634.

11 S. A. Bonnett, J. R. Whicher, K. Papireddy, G. Florova, J. L. Smith and K. A. Reynolds, Chem. Biol., 2013, 20, 772. 